\title{
Análisis de la proteína hidrolizada extraída del tejido de la curvina y la tilapia en el alimento del camarón
}

Edgar Osiris Carranza ${ }^{1}$

Rulbin Ariel Velásquez ${ }^{2}$

Ariel Fernando Rivas ${ }^{3}$

\section{RESUMEN}

La industria del camarón en Honduras registra un área de producción de 22,748 hectáreas que demandan un consumo anual de 80,000 toneladas métricas de alimento balanceado, este es un insumo de producción con alto costo para el productor. Durante el cultivo, peces como la curvina y la tilapia que crecen simultáneamente con el camarón y por su pequeño tamaño en la cosecha del camarón estas especies no son aprovechados, se considera que uno de los métodos más económicos para aprovechar estos recursos es el hidrolizado de pescado. El objetivo del estudio fue evaluar el efecto del hidrolizado de la curvina y la tilapia en el alimento balanceado sobre el desempeño productivo del camarón. Se usaron nueve acuarios de $37 \mathrm{~L}$, sembrando 12 camarones con pesos iniciales de $3.6 \mathrm{~g}$, las proteínas hidrolizadas fueron extraídas de tejidos de peces de curvina y tilapia. Se manejaron tres tratamientos que consistieron en a.) alimentar los camarones con el alimento balanceado más el extracto hidrolizado de la curvina, b.) alimento balanceado más el hidrolizado de tilapia y c.) solo alimento balanceado. Cada tratamiento fue manejado en una proporción triplicada. La mayor atractabilidad se observó en los camarones alimentados con el balanceado más el hidrolizado de tilapia $(P<0.0001)$. Los camarones son más voraces en horas de la mañana que por la tarde. Los crecimientos, factor de conversión alimenticia y tasa específica de crecimiento $(P<0.001)$ fueron más favorables en los animales alimentados con el hidrolizado de tilapia. Las mejores supervivencias se observaron en los camarones que consumieron los hidrolizados de pescado.

Palabras clave: hidrolizado de pescado, alimento balanceado, desempeño productivo.

\footnotetext{
${ }^{1}$ Profesor del Departamento de Acuicultura y Biología Marina, Centro Regional Universitario del Litoral Pacífico, UNAH: edgar.carranza@unah.edu.hn

2 Jefe de sistemas de gestión, Grupo Náutico: rulbinvelasquez@gmail.com

${ }^{3}$ Asistente Técnico de producción, Empresa ICASUR: arielrivas92@gmail.com
} 


\section{ABSTRACT}

The Honduran shrimp industry registers a production area of 22,748 hectares that demand an annual consumption of 80,000 metric tons of feed; this is a production input with a high cost to the producer. During the culture, fish such as corvine and tilapia grow simultaneously with shrimp and because of their size are not used. It is considered that one of the most economical methods to take advantage of these resources is fish hydrolysate. The objective of the study was to evaluate the effect of curvina hydrolysate and tilapia on the balanced feed of the productive performance of shrimp. Nine $37 \mathrm{~L}$ aquariums were installed planting 12 shrimp (with initial weight of $3.6 \mathrm{~g}$ ), hydrolyzed proteins were extracted from curvine and tilapia tissues, and three treatments were used. The treatments consisted in a.) feeding the shrimp with the balanced food plus the curly hydrolyzed extract, b.) feeding balanced food plus tilapia hydrolysate and c.) feeding only balanced food. Each treatment was managed in triplicate proportions. The greater attractiveness was observed in the shrimp fed with the balance plus the tilapia hydrolysate $(P<0.0001)$. The shrimp are more voracious in the morning than in the afternoon. The growths, feed conversion factor and specific rate of growth $(P<0.001)$ were more favorable in the animals fed tilapia hydrolysate. The best survivals were observed in the shrimp that consumed the fish hydrolysates.

Keywords: attractiveness, fish hydrolysate, balanced feed, productive performance. 


\section{INTRODUCCIÓN}

La industria del camarón en el año 2015 generó más de 177 millones de dólares americanos por la exportación de 56.81 millones de libras camarón en Honduras $(\mathrm{BCH}$, 2016), el área registrada de producción es de 22,748 hectáreas que se cultivan hasta tres veces por año (ANDAH, 2015). Esta producción demanda un consumo anual que supera las 80,000 toneladas métricas de alimento balanceado con alto valor proteínico.

Para los productores de camarón el alimento balanceado es un insumo de producción con un costo muy elevado, ya que la harina de pescado como la fuente principal de materia prima depende del valor del mercado internacional con tendencia a la alza (Figueiredo, 2016). El consumo para un productor en promedio es 1.4 toneladas métricas de alimento por hectárea por ciclo de cultivo, que representa un poco más de U\$D 1,148.00, haciendo insostenible la actividad productiva para muchos acuicultores por el volumen de alimento que se consume y su precio (Oyuela, 2016).

Los fabricantes de alimento balanceado adicionan suplementos como probióticos, prebióticos, antibióticos, enzimas, pigmentos, preservantes, atractantes y estimuladores del apetito que aceleran el crecimiento en los animales (Peregrin, 2013). También para optimizar el costo de fabricación de un balanceado, usan fuentes de proteína vegetal como sustituto de la proteína animal, esta proteína es de menor calidad al no contar con todos los aminoácidos esenciales que el camarón necesita (Pohlenz, 2016). Para lograr un óptimo cultivo se debe satisfacer todos los requerimientos nutricionales del camarón.

Durante el ciclo de producción existe fauna acompañante como la sardina (Lile stolifera, Anchoa sp.), curvina (Cynoscion sp.) la bubucha (Gambusia sp.) que crecen compitiendo con el cultivo en el estanque, y la tilapia (Oreochromis sp.) que se observa en los canales reservorios, todas estas especies consumen oxígeno, alimento y liberan desechos metabólicos que contribuyen en alterar la calidad del agua para el cultivo del camarón. Al momento de la cosecha estos organismos representan un volumen de la biomasa que no es aprovechada por su tamaño que no alcanza una talla comercial y podría servir como fuente alternativa de proteína de calidad y a bajo costo en la dieta del camarón. Proteína que tiene alta concentración de aminoácidos esenciales (AAE) y con alto valor nutritivo (National Research Council, 2011).

Los peces y los camarones necesitan proteínas de alta digestibilidad en cantidad y 
calidad. Tantikitti (2014) demostró que las ganancias de peso de los camarones están relacionadas con la cantidad y digestibilidad de la proteína. La lisina, metionina y cistina son los AAES más importantes en las dietas de camarones. La arginina es antagónica con la lisina y en niveles excesivos causa depresión en el crecimiento. La curvina y la tilapia son peces que cuentan con buena disposición de AAES en su organismo, y puede considerarse una fuente natural de proteínas (Gónzales, Maafs, \& Galindo, 2013).

Sin embargo, la inclusión de estos ingredientes alternativos en una dieta balanceada no es factible por el proceso de industrialización de esta proteína en forma de harina que para el productor su procesamiento sería en forma artesanal, reduciendo el valor nutricional y la calidad del alimento (Galano \& Farnés, 2015). Siendo el uso de la inclusión de la proteína hidrolizada sobre la dieta balanceada una alternativa para aprovechar estos recursos y optimizar la rentabilidad en los cultivos.

Las enzimas proteólicas comerciales provienen de origen vegetal y microbiológico como la papaina, la bromelina, Bacillus sp. y Aspergilllus sp., estás actúan como enzimas exopeptidasas y endopeptidasas o ambas rompiendo los enlaces peptídicos a péptidos más pequeños y mezclas de aminoácidos libres (Ramírez, 2010). Estas son usadas en la industria alimenticia como extractos de la proteína en los mariscos y pescado. El beneficio es mejorar la calidad del producto terminado, incrementar la digestibilidad y disponibilidad de la proteína hasta un $20 \%$, y de estas manera reducir el contenido de lípidos y cenizas (Molla \& Hovannisyan, 2011). Las enzimas proteolíticas mejoran el sabor del alimento y el color, es utilizado en pescados, filetes, langostas, cangrejos y camarones.

La hidrolisis enzimática de proteínas de pescado o proteína hidrolizada es la descomposición de la materia orgánica en otra forma más sencilla, son proteínas que degradan enzimáticamente péptidos de diferentes tamaños, aumentan el valor nutritivo de las dietas con poca proteína y son usados como atractantes que incrementan el consumo, reducen la pérdida del alimento y son absorbidas en el intestino sin una previa digestión en el estómago (Benitez, Ibarz, \& Pagan, 2008). Además, tienen propiedades con la activación y promoción de enzimas digestivas, incremento del peristáltico intestinal y los aminoácidos libres pueden funcionar como atrayentes (Gallardo, Pedroza, Brito, Cuzón, \& Gaxiola, 2004).

Los camarones son organismos omnívoros, en los primeros estadíos el sistema digestivo sufre transformaciones con cambios digestivos, bioquímicos y de comportamiento, esto los adapta para que el resto de su vida puedan alimentarse con microal- 
gas, zooplancton, materia orgánica, bacterias que se encuentran en el fango y restos de animales muertos como peces y crustáceos con los que presenta una alta atractabilidad (Pérez, Cruz, Abramo, \& Vega, 2006). Se desconoce el efecto las proteínas hidrolizadas de la curvina y la tilapia en el alimento balanceado como atractante alimenticio sobre el crecimiento del camarón.

El objetivo de este estudio fue evaluar el efecto del extracto hidrolizado de los tejidos de la curvina y la tilapia en el alimento balanceado para optimizar el consumo y el desempeño productivo del camarón durante 49 días de cultivo, y de esta manera determinar la mayor atractabilidad de los camarones en el alimento balanceado con la adición de proteínas hidrolizadas de tejidos de la curvina y la tilapia, así como comparar los indicadores de desempeño (crecimiento, factor de condición, factor de conversión alimenticia) y las supervivencias.

\section{MATERIALES Y MÉTODOS}

El estudio se realizó en el laboratorio húmedo del Centro Universitario Regional del Litoral Pacífico (CURLP) ubicada en el departamento de Choluteca en los meses de febrero a mayo del año 2016 bajo condiciones controladas. El lugar registra $1855 \mathrm{~mm}$ de precipitación, $28^{\circ} \mathrm{C}$ de temperatura promedio anual y se ubica a $31 \mathrm{msnm}$.

Para el estudio se utilizaron 108 camarones con un peso inicial de $3.64 \mathrm{~g}$. que fueron capturados de una finca de cultivo ubicada a $42 \mathrm{Km}$ al sur oeste del CURLP, se transportaron en condiciones controladas y se aclimataron en un tanque de 1000 litros de agua durante 48 horas, posteriormente se sembraron 12 animales por acuarios con un volumen de 37 litros. Durante todo el estudio se usó agua filtrada tratada en un laboratorio de producción de post-larva. Se suministró aireación constante y se realizaron recambio total de agua cada cinco días. Diariamente se registraron tres veces los parámetros de oxígeno disuelto (OD), temperatura, $\mathrm{pH}$ y salinidad.

\section{Diseño del experimento}

Se usaron nueve acuarios y se manejaron tres tratamientos. Los tratamientos consistieron en el suministro del alimento balanceado al $30 \%$ PC más la inclusión del extracto de la proteína hidrolizada de los tejidos de pescado. El primer tratamiento (T1) consistió en alimento balanceado más un baño de proteína hidrolizada de tejido de curvina; el segundo tratamiento (T2) fue el alimento balanceado más un baño de 
proteína hidrolizada de tejido de tilapia; y el tercer tratamiento (T3) consistió en alimentar solo con el alimento balanceado. Cada tratamiento fue manejado por triplicado. Los camarones se alimentaron con el $3 \%$ del peso vivo, y se fraccionó la ración diaria en un $40 \%$ a las 8 am y $60 \%$ a las 5 pm.

Para la preparación de los hidrolizados se usaron $500 \mathrm{~g}$ de filete de cada pescado (tilapia y curvina), se lavaron con abundante agua y se cortaron en trozos pequeños, seguidamente se introdujeron en un recipiente donde se les adicionó $500 \mathrm{~g}$ de agua y $1 \mathrm{~g}$ de la enzima proteolítica. El producto comercial que se usó como enzima fue el Protamex (Bacillus subtilis). La mezcla se calentó a $55^{\circ} \mathrm{C}$ durante 45 minutos para activar la enzima e iniciar el proceso hidrolítico. Posteriormente se volvió a calentar a $90^{\circ} \mathrm{C}$ por 45 minutos para finalizar el proceso, y se dejó en reposo por 15 minutos para inactivar la enzima. La mezcla final fue filtrada para separar los sólidos restantes y el sobrenadante se recolecto en frascos de vidrio que se cerraron herméticamente, se rotularon y se almacenaron a $5^{\circ} \mathrm{C}$.

Para preparar el alimento con los hidrolizados, las raciones fueron humedecidas al $15 \%$ de su peso con los extractos hidrolizados cada vez que se alimentaba. Para evitar contaminación de los extractos por la manipulación, diariamente se recolectaba en otros frascos de vidrio la cantidad de muestra que se necesitaría para tratar a los alimentos balanceados.

\section{Intervenciones}

Se analizó la atractibilidad, es decir, la atracción del alimento por parte de los camarones, registrando el tiempo de reacción en segundos (s) de los animales para capturar y engullir el alimento. Para obtener los indicadores de desempeño se analizó el crecimiento semanal, el factor de conversión alimenticia (FCA), la tasa especifica de crecimiento (TEC) y la sobrevivencia semanal. Se registró el peso promedio los camarones de cada acuario una vez por semana, la cantidad de alimento proporcionado, y los animales vivos y muertos de los acuarios.

Para calcular el FCA, TEC y la sobrevivencia se manejaron las siguientes formulas:

Ganancia de peso semanal $=B T C / N^{\circ}$ de camarones

Factor de conversión alimenticia $=$ TAS/BTC

Tasa especifica de crecimiento $(\% /$ día $)=100^{*}(\mathrm{LnPf}-\mathrm{LnPi}) / \mathrm{t}$

Supervivencia $(\%)=100^{*}(\mathrm{Cv} / \mathrm{Csi})$

Donde:

Pf=peso promedio final; 
$\mathrm{Pi}=$ peso promedio inicial;

TAS = total de alimento suministrado;

BTC = biomasa total de los camarones;

$t=$ tiempo de duración en días del cultivo;

LnPf=logaritmo natural del peso corporal promedio final;

$\mathrm{LnPi}=$ logaritmo natural del peso corporal promedio inicial;

$\mathrm{Cv}=$ camarones vivos al final del experimento;

Csi=camarones sembrados inicialmente;

\section{Análisis estadístico}

Se analizó la atractabilidad de los extractos hidrolizados a través de un diseño factorial de $3 \times 3 \times 2 \times 7$ (tres tratamientos, tres repeticiones, dos tiempos diarios de medición, siete semanas). En las comparaciones de los indicadores de desempeño como crecimientos, factor de conversión alimenticia y sobrevivencia, se usaron diseños factoriales de $3 \times 3 \times 7$ (tres tratamientos, tres repeticiones y siete semanas). En el análisis de las sobrevivencias los valores porcentuales fueron transformados con la ecuación arcoseno $\sqrt{ }(y \mathbf{y}(\%) / 100)$ para potenciar el análisis de la varianza y la comparación múltiple de medias.

En la comparación múltiple de medias de usó la prueba de la Diferencia Mínima Significa (DMS) al 0.05 de error. Para análisis de los datos se manejó el programa estadístico InfoStat@ versión 2015.

\section{RESULTADOS}

\section{Atractabilidad}

Se observaron diferencias altamente concluyentes entre los tres tratamientos $(P<0.0001)$ encontrando el menor tiempo de captura e ingesta del alimento en la ración tratada con el extracto hidrolizado de tilapia con 9.5 segundos, seguido por el extracto hidrolizado de la curvina con 26.7 segundos y con 61.8 segundos el alimento balanceado. La mayor atractabilidad se observó en horas de la mañana $(P<0.0001)$ encontrando el menor tiempo de captura en las raciones tratadas con el extracto hidrolizado de tilapia (Cuadro 1). 


\section{Cuadro 1. Atractabilidad de camarones por alimento balanceado tratado con extractos hidrolizados de pescado}

\begin{tabular}{|l|c|r|l|}
\hline \multicolumn{1}{|c|}{ Tratamiento } & $\begin{array}{c}\text { Hora de } \\
\text { alimentación }\end{array}$ & $\begin{array}{c}\text { Tiempo de } \\
\text { reacción en s }\end{array}$ \\
\hline Alimento + hidrolizado de tilapia & Mañana & 7.6 & a \\
\hline Alimento + hidrolizado de tilapia & Tarde & 11.3 & a \\
\hline Alimento + hidrolizado de curvina & Mañana & 14.6 & b \\
\hline Alimento + hidrolizado de curvina & Tarde & 39.8 & C \\
\hline Alimento & Mañana & 43.5 & C \\
\hline Alimento & Tarde & 80.4 & C \\
\hline Medias con una letra común no son significativamente diferentes $(p>0.05)$ & \\
\hline
\end{tabular}

Los camarones alimentados sin los hidrolizados de pescado, presentaron el mayor tiempo de captura y engullición del alimento.

\section{Indicadores de desempeño}

En los crecimientos semanales se encontraron diferencias altamente concluyentes entre tratamientos $(P<0.001)$, el hidrolizado de tilapia presentó la mayor ganancia de peso con $0.94 \mathrm{~g}$ a la semana, con el extracto de la curvina se obtuvo $0.77 \mathrm{~g}$ y con el alimento balanceado se logró $0.65 \mathrm{~g}$. Al final del estudio los pesos finales en promedio fueron de $10.1 \mathrm{~g}$ en los camarones tratados con el extracto hidrolizado de la tilapia, $9.0 \mathrm{~g}$ para los tratados con el hidrolizado de curvina y $8.15 \mathrm{~g}$ los alimentados con balanceado (Figura 1). Los camarones alimentados con el extracto hidrolizado de tilapia crecieron hasta un $24 \%$ más que los alimentados con el balanceado, y los camarones que se alimentaron con el hidrolizado de curvina su crecimiento fue $11 \%$ más que los alimentados con la dieta balanceada.

En la comparación del Factor de Conversión Alimenticia (FCA) se encontraron diferencias altamente significativas $(P<0.0001)$, la mejor eficiencia de conversión al final del estudio se observó en los camarones alimentados con el extracto hidrolizado de tilapia con 1.65, seguido por los camarones alimentados con el extracto hidrolizado de la curvina con 2.11 y el de menor eficacia en conversión fueron los camarones alimentados con balanceado con 2.80. El FCA indica la cantidad de alimento en $\mathrm{Kg}$ que los animales consumieron por cada $\mathrm{Kg}$ ganado en peso, siendo las dietas más eficientes las que presentan tasas de conversión más bajas. 
Figura 1. Crecimiento semanal promedio de camarones alimentados con dieta balanceada y extracto hidrolizado de pescado durante siete semanas de cultivo

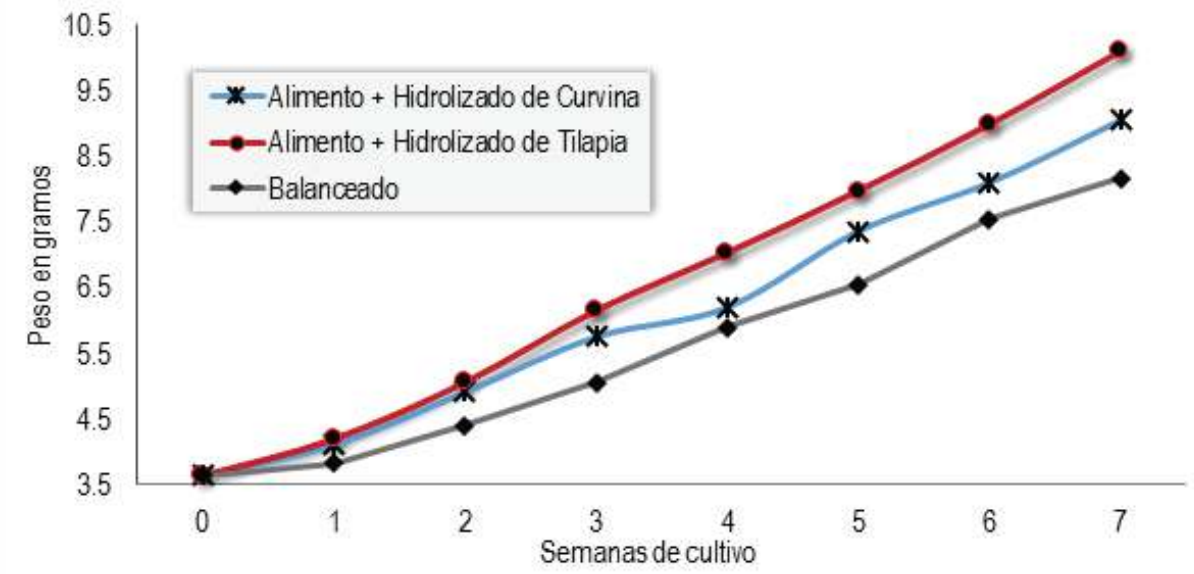

En la tasa específica de crecimiento se observaron valores concluyentes $(P<0.0001)$ con los resultados más favorables para los animales que fueron alimentados con el extracto hidrolizado de tilapia. Durante las siete semanas de estudio las mejores TEC semanales se encontraron en los camarones tratados con el extracto hidrolizado de tilapia (Figura 2), en el caso de los camarones alimentados con el extracto de la curvina la TEC semanal fue más variable, pero se observó valores por encima de los camarones alimentados con la dieta balanceado sin la inclusión de los hidrolizados de pescado. Al final del estudio la TEC fue de 1.87 en los camarones tratados con hidrolizados de pescado, 1.58 en los camarones alimentados con el hidrolizado de la curvina y 1.11 en los animales alimentados con la dieta balanceada.

Figura 2. Tasa específica de crecimiento en camarones alimentados con dieta balanceada y extracto hidrolizado de pescado durante siete semanas de cultivo

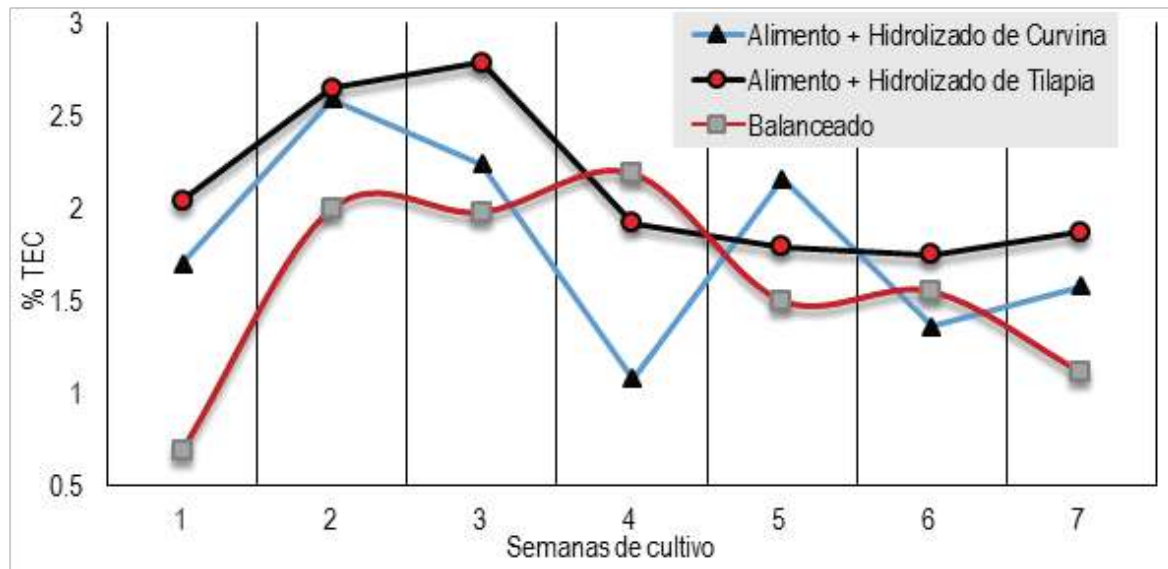


La TEC indica el grado de crecimiento diario de los camarones expresados en porcentaje, los animales alimentados con el extracto hidrolizado de tilapia presentaron TEC entre 2.79 y $1.87 \%$ en promedio por semana, 2.5 y $1.3 \%$ en los camarones alimentados con el extracto hidrolizado de curvina, y entre 2.11 y $0.69 \%$ en los animales alimentados con la dieta balanceada respectivamente.

\section{Supervivencias}

En las supervivencias se encontraron diferencias concluyentes entre los tratamientos $(P<0.0001)$, las mayores supervivencias en promedio se observaron en los camarones que se alimentaron los hidrolizados de pescado, la supervivencia más baja se presentó en los animales que consumieron solo la dieta balanceada. Entre semanas, las mejores supervivencias se encontraron en las primeras tres semanas, al incrementar los días de estudio del experimento las supervivencias se redujeron (Figura 3) en todos los acuarios.

Figura 3. Porcentaje de sobrevivencia en camarones alimentados con dieta balanceada y extracto hidrolizado de pescado durante siete semanas de cultivo

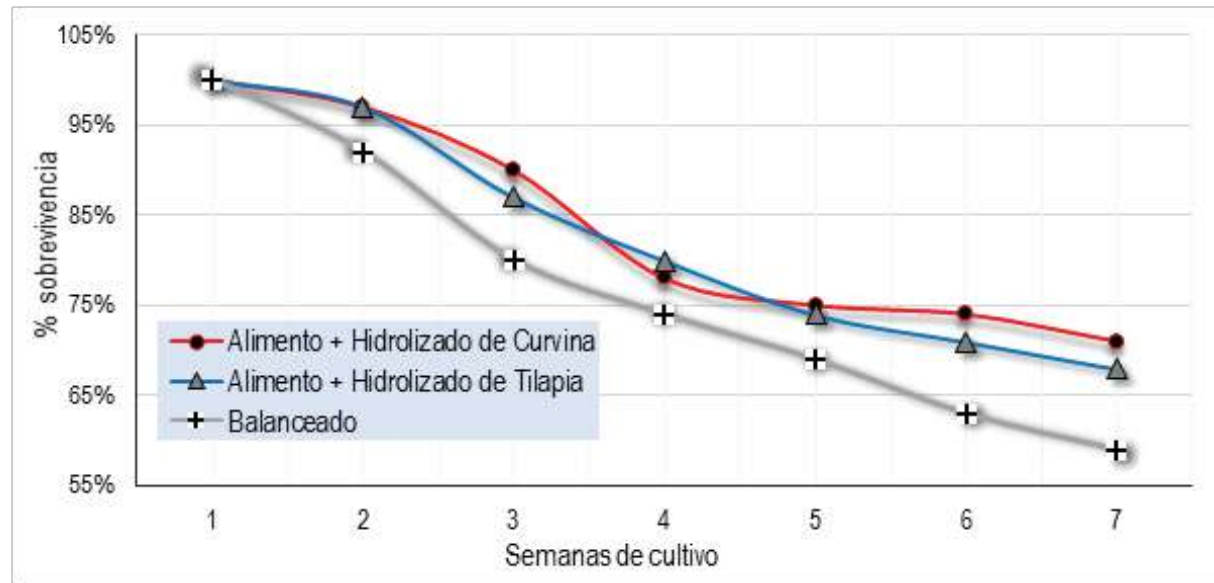

La sobrevivencia final fue de $71 \%$ para los camarones alimentados de con el extracto hidrolizado de curvina, seguido por los animales tratados con el extracto hidrolizado de tilapia con $68 \%$, y $52 \%$ para los camarones alimentos únicamente con el balanceado. Estadísticamente no se encontraron resultados concluyentes entre la sobrevivencia final de los camarones alimentados con los hidrolizados de pescado. 


\section{DISCUSIÓN}

Los hidrolizados de pescado incrementaron la atractabilidad en los camarones con la captura del alimento, estos se sintieron atraídos por disponibilidad de la proteína animal y sobre todo por sus hábitos alimenticios naturales al ser necrófagos. También los extractos hidrolizados de las proteínas de pescado potenciaron el sabor del alimento balanceado por la alta cantidad de aminoácidos libres presentes en los hidrolizados (Ramírez, 2010). Los camarones alimentados sin los hidrolizados reaccionaron en el tiempo esperado con 43.5 segundos por la mañana y 80.4 segundos en la tarde, las dietas balanceadas para camarones se fabrican para que sean consumidos y mantengan sus propiedades físicas (permeabilidad e hidroestabilidad) por más de 240 minutos una vez sumergidos en el agua (Sandoval, 2011). Para los productores ese tiempo asegura el consumo del alimento por parte del camarón.

En los crecimientos finales y semanales, los camarones alimentados con el hidrolizado de tilapia presentaron mayor ganancia de peso, seguido por los alimentados con el hidrolizado de curvina, estas diferencias se debe a la cantidad de aminoácidos libres y disponibles en los hidrolizados de los pescados (Cuadro 2) sugerido por Izquierdo, Torres, Allara, Márquez, \& Barbosa (2001), sobresaliendo el tejido de la tilapia con mayor cantidad de proteína y mayor cantidad de aminoácidos totales.

Los camarones tienen una capacidad limitada para sintetizar proteínas y logran crecimientos cuando lo adquieren del alimento que se consumen (Molina, 2015). Los aminoácidos tienen funciones específicas en los camarones, AAES como la isoleucina promueve crecimiento y el equilibrio de nitrógeno en el cuerpo y sintetiza otros aminoácidos no esenciales, la leucina aumenta los niveles musculares de energía, la lisina sirve para la construcción de anticuerpos de la hemolinfa que fortalece el sistema circulatorio, la metionina metaboliza las grasas y lípidos, y mantiene la salud del hepatopáncreas, la fenilalanina estimula el metabolismo, así como la treonina mejora la capacidad intestinal y la asimilación digestiva (Damal, y otros, 2013). Los niveles superiores de AAES en los hidrolizados de tilapia mejoraron el desempeño en los camarones con la ganancia de peso.

En la tasa específica de crecimiento los resultados fueron más favorables para los camarones alimentados con el extracto hidrolizado de tilapia, los valores de la TEC disminuyeron a medida que los camarones fueron creciendo y consumiendo más alimento (Cho \& Talbot, 2016). En acuacultura la TEC es usada para proyectar los crecimientos en tiempos determinados. 
Cuadro 2. Análisis proximal y composición de aminoácidos esenciales de los tejidos de curvina y tilapia

\begin{tabular}{|c|c|c|}
\hline $\begin{array}{c}\text { Aminoácidos } \\
\text { esenciales }\end{array}$ & curvina & tilapia \\
\hline Histidina & 1.222 & 1.625 \\
\hline Treonina & 0.442 & 0.807 \\
\hline Tirosina & 1.567 & 1.898 \\
\hline Metionina & 0.271 & 0.555 \\
\hline Valina & 0.847 & 1.413 \\
\hline Fenilalanina & 0.716 & 1.117 \\
\hline Isuleucina & 0.952 & 1.583 \\
\hline Leucina & 1.017 & 1.454 \\
\hline Lisina & 1.489 & 0.486 \\
\hline Total, de aminoácidos & 8.568 & 10.938 \\
\hline Proteína & 20.73 & 23.34 \\
\hline Grasa & 2.11 & 2.26 \\
\hline Cenizas & 1.90 & 1.94 \\
\hline \% Humedad & 76.95 & 72.36 \\
\hline
\end{tabular}

Fuente: (Izquierdo, Torres, Allara, Márquez, \& Barbosa, 2001)

\section{CONCLUSIONES}

La mayor atractabilidad de los camarones se encontró en el extracto hidrolizado de la tilapia $(P<0.0001)$, el tiempo de reacción de los animales para capturar el alimento fue de 9.5 segundos en promedio. En general los camarones tienen mayor voracidad en la jornada de la mañana con 21 segundos para capturar el alimento.

Los camarones alimentados con el extracto hidrolizado de la tilapia fue el que presentó el mayor crecimiento con $0.92 \mathrm{~g}$ de ganancia de peso corporal a la semana. En comparación con el tratamiento del alimento balanceado, los camarones crecieron hasta un $24 \%$ más con el extracto hidrolizado de tilapia y un $11 \%$ más con el hidrolizado de curvina.

En la tasa específica de crecimiento el hidrolizado de tilapia mostró hasta un $2.0 \%$ en promedio de crecimiento diario. En el FCA el extracto hidrolizado de tilapia presentó 
las mejores conversiones con 1.65 en comparación con el alimento balanceado que fue de 2.80, logrando un mayor aprovechamiento del alimento.

Las mejores sobrevivencias se lograron en los tratamientos donde se adicionó extractos hidrolizados de proteínas $(p<0.001$ ) con 68 y $71 \%$ de sobrevivencias respectivamente. La sobrevivencia más baja se encontró en el tratamiento de alimento balanceado.

\section{REFERENCIAS BIBLIOGRÁFICAS}

ANDAH. (02 de septiembre de 2015). Asociación Nacional de Acuicultores de Honduras. Recuperado de Noticias: Sin legalizar 142 fincas camarones: http://www.andah.hn/ver/feed/14/

BCH. (febrero de 2016). Banco Central de Honduras. Recuperado el 02 de enero de 2017, de Exportaciones FOB de Mercancías Generales 2000-2015: http://www.bch.hn/exportaciones.php

Benitez, R., Ibarz, A., \& Pagan, J. (2008). Hidrolizados de proteínas, procesos y aplicaciones. Acta Bioquímica Químia Latinoamericana, 42(2), 228-236. Recuperado de https://www.researchgate.net/publication/262552066_Hidrolizados_de_proteina_Procesos_y_aplicaciones

Cho, Y., \& Talbot, C. (30 de mayo de 2016). Señor Salmon. Recuperado de SGR y GF3 formas de estimar el crecimiento en nuestros peces: http://senorsalmon.blogspot.com/2016/05/sgr-y-gf3-formas-de-estimar-el.html

Damal, J. S., Ponniah, A., Khan, H. I., Babu, E. M., Ambasankar, K., \& Kumarguru, K. (junio de 2013). Shrimps, a nutritional perspective. Current Sciencie, 104(11), 1487-1491.

Figueiredo, C. (2016). XI Simposio Centroamericano de Acuícultura ANDAH. Nuevos avances en la nutrición del camarón para la producción de alimentos sustentables (págs. 12-16). Choluteca: ANDAH.

Galano, T. G., \& Farnés, O. C. (julio de 2015). Nutrición del camarón blanco, 25 años de investigación cientifica. (U. d. Centro de Investigaciones Marinas, Ed.) Investigaciones Marinas, 35(2), 24-40. Recuperado de http://www.cim.uh.cu/rim/

Gallardo, P., Pedroza, R., Brito, A., Cuzón, G., \& Gaxiola, G. (2004). Memorias del VII Simposio Internacional en Nutrición Acuícola. Uso de Alimentos Artificiales con Hidrolizados Proteícos de Origen Marino en Larvicutura de Camarones, Efecto Sobre la Respuesta Nutrimental y Balance Energético (págs. 145-167). Hermosillo, Sonora: Avances en Nutrición Acuícola.

Gónzales, M. I., Maafs, A. G., \& Galindo, C. (diciembre de 2013). Perfil de ácidos grasos de diversas especies de pescados consumidos en México. Biologica Trópical, 61(4), 1981-1998. 
Izquierdo, P., Torres, G., Allara, M., Márquez, E., \& Barbosa, Y. (2001). Análisis proximal, contenido de aminoácidos esenciales y relación calcio-fósforo en algunas especies de pescado. Revista Científica FCV-LUZ, XI(2), 95-100. Recuperado de http://www.saber.ula.ve/bitstream/123456789/27453/2/articulo2.pdf

Molina, C. A. (2015). Evaluación de varias fuentes de protíena vegetal en dietas para camarón. Tesis de doctorado, Universidad Politecnica de Valencia, Departamento de Ciencia Animal, Valencia. Recuperado de https://riunet.upv.es

Molla, A. E., \& Hovannisyan, H. G. (2011). Optimization of enzymatic hidrolysis of visceral waste proteins of beluga Huso huso using Protamex. International Aquatic Research, 93-99.

National Research Council. (2011). Nutrien and requirement of fish and shrimp. Washington, D.C., EEUU: National Academies Press.

Oyuela, R. (23 de mayo de 2016). Costo y consumo mínimo de alimento balanceado en un ciclo de cultivo de camarón. (E. O. Carranza, Entrevistador) Choluteca, Choluteca.

Peregrin, E. (junio de 2013). Nuevas alternativas de dietas de bajo costo para el cultivo del camarón en Cuba. Revista electrónica de Veterinaria, 14(6), 1-7. Recuperado de http://www.veterinaria.org/revistas/redvet/n060613/061304.pdf

Pérez, I., Cruz, P., Abramo, D., \& Vega, H. G. (abril de 2006). Evaluación de la actividad enzimatica y contenido de proteínas en camarón blanco alimentado con diferentes dietas. Revista Electrónica Veterinaria, II(4), 22-35. Recuperado de http://www.veterinaria.org/revistas/redvet/n040406.html

Pohlenz, C. (2016). XI Simposio Centroamericano de Acuacultura ANDAH. Nutrición aplicada en busca del mejor rendimiento económico (págs. 22-28). Choluteca: ANDAH.

Ramirez, D. C. (2010). Obtención de hidrolizado de proteína de pescado a partir de la tilapia roja (Oreochromis sp.). Universidad Nacional de Colombia. Bogotá: Facultad de Ciencias. Recuperado de http://www.bdigital.unal.edu.co/2786/1/107411.2010.pdf

Sandoval, M. J. (2011). Parametros operacionales para la eficiencia de la producción y calidad de alimentos balanceados en pellet para camarón. Universidad de San Carlos de Guatemala, Escuela de Ingeniería. Guatemala: Facultad de Ingeniería. Recuperado de http://biblioteca.usac.edu.gt/tesis/08/08_1208_Q.pdf

Tantikitti, C. (enero de 2014). Feed palatability and the alternativa protein sources in shrimp feed. (P. o. Departament of Aquatic Science, Ed.) Songklanarakin Journal of Science and Technology, 36(1), 51-55. Recuperado de http://rdo.psu.ac.th/sjstweb/ 